\title{
Modeling of reconfigurable manufacturing systems for determining their performance
}

\author{
Alexey Kapitanov ${ }^{1, *}$, and Vladimir Mitrofanov ${ }^{1}$ \\ ${ }^{1}$ Moscow State Technological University «Stankin», Vadkovskiy lane, 1, Moscow, 127055, Russian Federation
}

\begin{abstract}
A method of determining flexible manufacturing system performance (throughput capacity) based on identifying elementary controlled events that occur during FMS operation and controlling this flow based on Shannon approach to evaluation of the quantity of information required for control is proposed. The behavior of the main FMS elements in the manufacturing process is suggested to be described using three types of random flows of square pulses - state, availability and propagation flows. The proposed mathematical model enables to choose the optimum relationship between FMS power margins, parts and tools supply and throughput capacity.
\end{abstract}

\section{Introduction}

Modeling is the most important tool while creating flexible manufacturing systems (FMS). The main types of models, applied to FMS shall be analytical and simulation ones (Table 1) [1-12].

The main goal of FMS control is the exact realization of the determined production plan. Where the qualitative indicators are represented by the maximum efficiency, implementability (coordination with production capacities), and information throughput capacity of control system required in the process of plan targets' implementation [13-18].

The last question for FMS control systems determines the conditions in which computer-aided control system (CACS) is put in the process of plan implementation. It may seem that the effect achieved with increase in the degree of optimality of the planning algorithm is completely offset by additional costs of computer-aided operational control system.

The determining of FMS performance (throughput capacity) is particularly important at its design stage. This requires the development of a model that adequately reflects the process and uses statistical data, available for relatively easy measurement on the operating FMS, as the source [19-24].

Regular elementary controlled events with different characteristics can be identified for FMS in mechanical engineering and instrument engineering, which are characterized by discreteness of products, consisting of multiple parts and assemblies, manufacturing facility, consisting of a number of machines with selected modes for moving and machining of products (various parts grouping for individual and mass manufacture).

Startup of batch manufacture and adjustment of the process of its passage through the facility during its machining refer to such events in FMS. The volume of data to be received and processed by CACS for FMS when controlling an elementary event is usually quite steady and depends on the number of alternatives considered in the decision-making process. Their number is determined by the batch type, number of parts in a batch, time interval between sequential startups and the nature of startup and release (scheduled, ahead of schedule). Shannon approach to evaluation of the quantity of information required for high quality control of elementary events' flow that occur in the process of FMS operation can be used with such choice of FMS elementary controlled events [25-29].

The elementary event control function involves synchronization of operation of the main elements of manufacturing process in accordance with the defined plan.

\section{Main part}

Behavior of the main FMS elements in the production process can be described using three types of random flows of square pulses, known as the flows of state, availability and propagation.

State flows $P(t)$ are generated from impulses with an amplitude of 1 , random pulse duration and pauses between them. They characterize the performance of equipment, attachments, apparatus, and control software etc. over time, and the duration of their recovery, and are determined by the laws of pulses and pauses duration distribution.

Availability flows $A(t)$ are generated by pulses with variable amplitude and duration, characterizing the and

\footnotetext{
* Corresponding author: av.kapitanov@stankin.ru
} 
Table 1. Main FMS Models

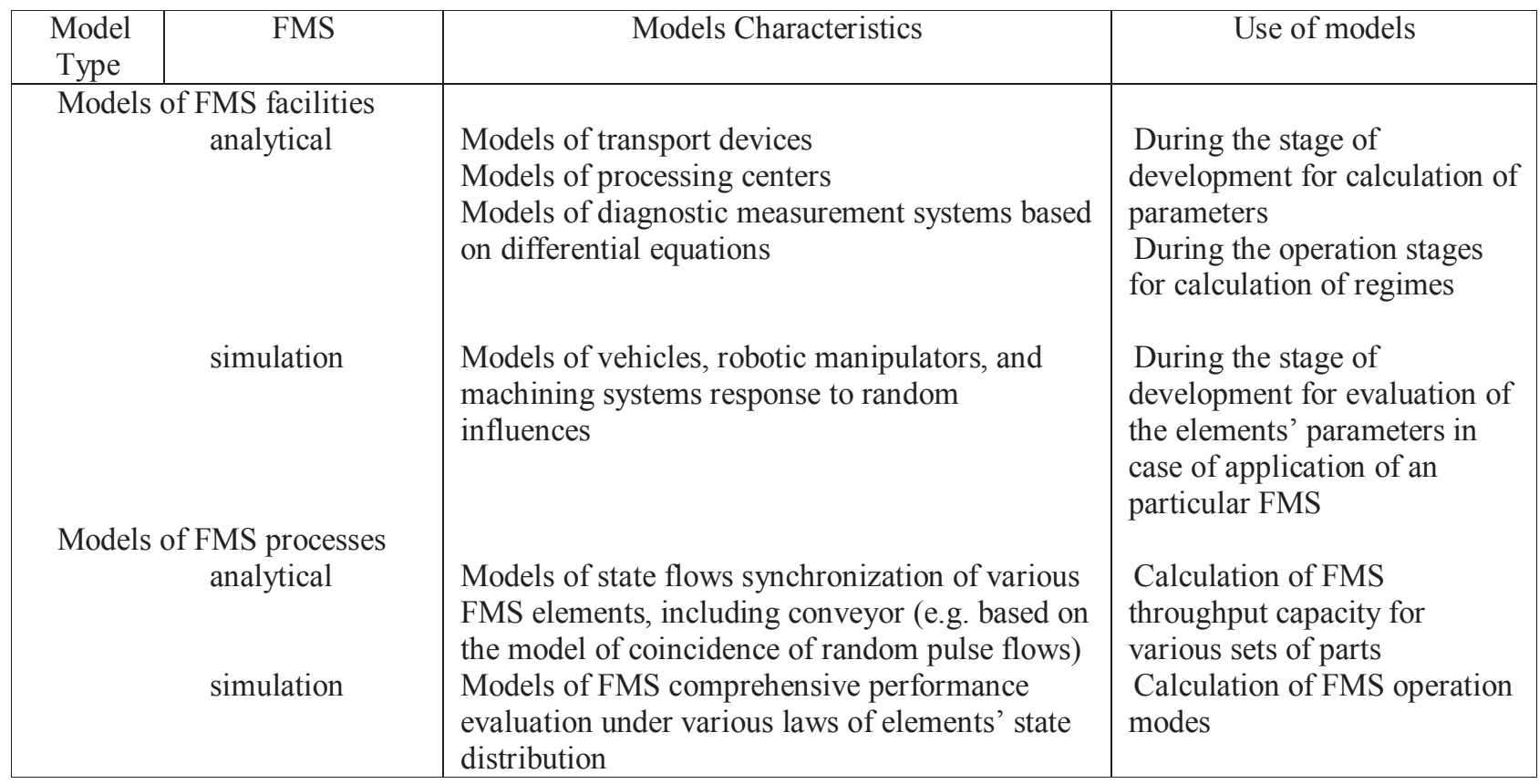

resources by units of equipment, they are determined by the laws of amplitudes' distribution and pulses duration.

Pulse propagation flows $B(t)$ characterize the flow of elementary controlled events passing through a particular equipment unit during machining. They are represented in two ways: as pulses with zero duration and variable amplitude, or as pulses with single amplitude and variable duration.

In both cases pulses have random repetition interval and variable parameters - either amplitude or duration, proportional to the number of parts that have been machined on this equipment unit.

Propagation flows are determined by the amplitudes' distribution laws, duration of pulses and pauses between them.

Synchronous behavior of production process elements is achieved by controlling, which obviously corresponds to time coincidence of random flows' pulse parameters characterizing elements that determine the possibility of implementation of this part operation on this equipment unit. The result is the coincidence flow that corresponds to the propagation flow of machined products through an equipment unit. Application of ncoincidence operation

$$
\mathrm{n}(\mathrm{t})=\underset{\mathrm{i}=1}{\mathrm{n}} \mathrm{P}_{\mathrm{i}}(\mathrm{t})
$$

is preceded by a number of additional operations: addition of the availability flow $A(t)$ to the propagation flow $B(t)$

$$
S_{k}\{A(t) ; B(t)\}=S_{k-1}\{A(t) ; B(t)\}+B\left(t_{k}\right)
$$

where $\quad t_{k}<t<t_{k+1}, \quad S_{0}\{A(t) ; B(t)\}=A(t) \quad$ the separation of the propagation flow from the availability flow pool (reserves) for different parts operations time

$$
f(t)=\min \{A(t) \vee B(t)\}
$$

conversion of the propagation flow into the state flow

$$
P(t)=\sum_{k=1}^{\infty}\left\{1\left(t-t_{k}\right)-1\left[t-t_{k}-B\left(t_{k}\right)\right]\right\} ;
$$

conversion of the state flow into the propagation flow

$$
\begin{aligned}
& B(t)=a(t) \int_{t_{k}-\tau_{k}}^{t_{k}} d t \\
& a(t)=\left\{\begin{array}{l}
0 \text { npu } t \neq t_{k} ; \\
1 \text { npu } t=t_{k},
\end{array}\right.
\end{aligned}
$$

where $t_{k}, \tau_{k}$ - are the random moments of state flow pulse occurrence and their duration; separation (subtraction) of the propagation flow from the availability flow $R_{k}\{A(t) ; B(t)\}=R_{k-1}\{A(t) ; B(t)\}-B\left(t_{k}\right)$

$$
\begin{aligned}
& \text { for } t_{k}<t<t_{k+1}, R_{0}\{A(t) ; B(t)\}=A(t) \text {; } \\
& B\left(t_{k}\right) \leq R_{k-1}\{A(t) ; B(t)\}
\end{aligned}
$$

The specified operations enable description of movement of the products' material flow (deliveries, rejects) and precede the time coincidence of single amplitude pulses $n(t)$. Further, the object of discrete manufacture is interpreted as discrete communication channel transmitting the propagation flow under noise conditions determined by parameters of state flows and $n$-coincidence operation. The actual and the required throughput capacity of the elementary facility (machine) control system can be determined based on the theorems of information theory, by calculating the entropy per 
elementary event of the source - $H(x)$ for all input flows, receiver - $H(y)$ for all output propagation flows (coincidence flow) and based on $n$-coincidence operation - conditional entropy $H_{x}(y)$. The actually provided throughput capacity per $\lambda$ equipment unit with $j_{\lambda}$ parts machined on it, and the startup intensity of production batches $N_{j \lambda} / T$

$$
C_{\lambda}=\max \frac{N_{j \lambda}}{T}\left[H(y)_{j \lambda}-H_{x}(y)\right]
$$

With simultaneously used $k$ equipment units the throughput capacity of FMS will be:

$$
C^{\prime}=\sum_{\lambda=1}^{k} C_{\lambda}
$$

Additionally required throughput capacity from CACS for FMS, attributed to one controlled event, can be determined in accordance with Shannon's 10th Theorem. Here, CACS for FMS is considered as corrector of the channel, which is FMS.

For the entire FMS with simultaneous use of $k$ machines the additionally required CACS for FMS throughput capacity

$$
\Delta C^{\prime}=\sum_{\lambda=1}^{k} \max \left\{\frac{\left.N \mid H(x)_{j \lambda}+H_{x}(y)_{j \lambda}-H(y)_{j \lambda}\right]}{T}\right\}
$$

at the same time the batch startup and release control require particular time regulated actions ensuring complete processing of information, decision-making and managerial activities.

\section{Conclusion}

Extension of FMS throughput capacity by means of CACS enables the implementation of a larger number of elementary controlled events at an average, while ensuring the required statistical parameters of the products propagation flow at FMS output.

The estimate obtained from (9) represents the lower limit of CACS for FMS throughput capacity extension in order to ensure the admissible parameters of $H(y)_{j \lambda}$ for all $\lambda$ and $j-C_{\min }=\left(C^{\prime}+\Delta C^{\prime}\right)$. The upper limit of throughput capacity extension $C_{\max }$ is determined from the ratio, connecting FMS resources involved in manufacture during control automation $E_{\tau}$ with difference between the source entropy value of the output flows $H_{x}(y)=H_{0}$ and the value achieved by extending CACS for FMS throughput capacity. This dependence can be determined as

$$
\left.E_{T}=E_{\max } \mid 1 \quad a_{1} e^{k_{i}\left[H_{0} H_{T}\right]}\right]
$$

and is similar to the dependence obtained by Academician V.A. Trapeznikov for complex manufacturing systems, at $H_{T_{1}} \leq H(y)_{a d d} C_{\max }$ is determined from the expression

$$
\left[\frac{d E_{T}}{d H_{T}}\right]_{a^{t} H_{T_{1}} \rightarrow \min } \geq \frac{\left.d|F(c)| H_{T_{1}}\right\rfloor}{{ }^{d} H_{T}}
$$

where $F(c)$ determines the costs of CACS for FMS depending on its throughput capacity. In this case FMS entropy is $-H_{T_{i}}$. The following relationship should be observed for efficiently designed system with optimum throughput capacity $\mathrm{C}_{\mathrm{opt}}$ :

$$
C_{\text {minat } L_{1} \rightarrow \text { var }}=H_{i}(x)\left[1 \quad a e^{{ }^{k} L_{1}}\right.
$$

In addition to FMS control automation, the increment of $\Delta C$ value can be ensured by increasing the mean amplitude value of availability flows (reserves, manufacturing capacity, and FMS equipment). $\Delta C$ value depends on the distribution of total resources between individual flows $L_{i}$. When $L_{i}$ is changed for one of the participants in $n$-coincidence of elementary flows operation

$$
\left.\Delta C_{\mid a t L_{i} \rightarrow \text { ara }}=H_{i}(x) \mid 1 \quad a e^{{ }^{k} L_{1}}\right]
$$

where $H(x)$ - if the input flow entropy; $a$ and $k$-are constants.

The use of (13) enables choosing the optimum relationship between FMS power margins, supply of components and tools and CACS for FMS throughput capacity.

The reported study was funded by RFBR, according to the research project No. 16-31-60079 mol_a_dk

\section{References}

1. H. ElMaraghy, International Journal of Flexible Manufacturing Systems Vol. 17(4), pp. 261-276 (2006)

2. G. Schuh, V.Stich Springer (2012)

3. F. Karl, G. Reinhart, M. Zaeh. 45th CIRP CMS, pp. 608-613 (2012)

4. D. Telgen, L. Moergestel, D. Ceglarek, Robotics and Computer-Integrated Manufacturing. Vol. 43, pp. 30-38 (2017)

5. V. Bryuhanov, Vestnik MSTU Stankin Vol. 2, pp. 56-62 (2010)

6. V. Voronenko, E. Mikhailov, Y. Sokolov, Vestnik MSTU Stankin Vol. 3 (34), pp. 29-33 (2015)

7. V. Mitrofanov, A. Kapitanov, A. Popov, ONIKS, (2013)

8. V. Chekanin, A. Chekanin, Information technology and computer systems. Vol. 2, pp. 37-45 (2014)

9. K. Mittal, P. Jain An. Procedia Engineering Vol. 69, pp. 1125-1129 (2014)

10. A. Azab, H. ElMaraghy, P. Nyhuis, J. PachowFrauenhofer, M. Schmidt, CIRP Journal of Manufacturing Science and Technology Vol. 6, pp. 110-119 (2013)

11. M. Bruccoleri, G. Lo Nigro, G. Perrone, P. Renna, 
S. Noto La Diega, Annals of the CIRP Vol. 54, pp. 433-436 (2005)

12. M. Abbasi, M. Houshmand, International Journal of Advanced Manufacturing Technology Vol. 54, pp. 373-392 (2011)

13. A. Tsarev, Problems of engineering and automation. Vol. 3, pp. 33-41 (2014)

14. B. Cherpakov, A. Feofanov, STIN Vol. 6, pp.3. (2004)

15. V. Merchants, A. Feofanov, Industrial and manufacturing engineering. Vol. 2, pp. 66-67 (2010)

16. T. Grishina, V. Mitrofanov, A. Feofanov, Vestnik of Belgorod state technological University n. a. V. G. Shukhov Vol. 3, pp. 158-160 (2014)

17. A. Feofanov, Y. Milkina, Economics and management in mechanical engineering Vol. 1, pp. 5-7 (2011)

18. A. Musatov, A. Apple, V. Kulakov, The scientific review Vol. 5, pp. 253-262 (2014).

19. R. Adgamov, S. Dmitriev, T. Karimov, A. Khayrullin, Scientific-technical Vestnik of the Volga region Vol. 6, pp. $72-75$ (2012).

20. B. Gorshkov, I. Samartsev, Vector of science of Togliatti state University. Vol. 3, pp. 53-56 (2014)

21. L. Shvartsburg, E. Butrimova, N. Drozdov, Safety Vol. 2, pp. 27-30 (2012)

22. L. Shvartsburg, Y. Zvenigorodsky, N. Bukeikhanov, Vestnik MSTU Stankin Vol. 2, pp. 14-17 (2011)

23. A. Kutin, M. Turkin, Vestnik MSTU Stankin Vol. 4, pp. 41-47 (2010)

24. A. Kutin, M. Turkin, Vestnik MSTU Stankin Vol. 3, pp. 36-40 (2011)

25. A. Ananiev, Vestnik MSTU Stankin Vol. 2, pp. 65$68(2010)$

26. A. Shabarov, Vestnik MSTU Stankin. Vol. 3, pp. 136-143 (2011).

27. V. Mitrofanov, T. Grishina, A. Feofanov, Industrial and manufacturing engineering Vol. 8, pp. 43-45 (2015)

28. A. Feofanov, O. Feofanov, I. Junín, Vestnik MSTU Stankin Vol. 4, pp. 95-99 (2009)

29. A. Kapitanov, Procedia Engineering Vol. 150, pp. $832-836(2016)$ 